\title{
Co-amplification of the HER2 gene and chromosome 17 centromere: a potential diagnostic pitfall in HER2 testing in breast cancer
}

Varga, Z ; Tubbs, R R ; Wang, Z ; Sun, Y ; Noske, A ; Kradolfer, D ; Bosshard, G ; Jochum, W ; Moch, $\mathrm{H}$; Öhlschlegel, C

\begin{abstract}
Co-amplification of the centromere on chromosome 17 (CEP17) and HER2 can occur in breast cancer. Such aberrant patterns (clusters) on CEP17 can be misleading to calculate the HER2/CEP17 ratio, and thus underreporting of HER2 amplification. We identified 14 breast cancers retrospectively with HER2/CEP17 co-amplification and performed FISH (fluorescence in situ hybridization) with additional chromosome 17 probes (17p11.1-q11.1, 17p11.2-p12, TP53 on 17p13.1, RARA on 17q21.1-3 and TOP2 on 17q21.3-22) to characterize the spanning of the amplicon in these cases. Furthermore, the HER2 status was analyzed by means of HER2 silver in situ hybridization (SISH) and immunohistochemistry (IHC). The co-amplification of HER2/CEP17 was compared between the three institutions. TP53 was eusomic in all cases, 17 p11.2-p12 in 79\% (11/14), whereas 17p11.1-q11.1 showed chromosomal gain in all cases. RARA was amplified in $10 / 14$ cases $(71 \%)$ and TOP2 in $3 / 14$ cases $(21 \%)$. HER2 was amplified with FISH/SISH in all 14 cases. $9 / 14$ tumors were $3+$ IHC positive $(64 \%)$ and 3 cases were $2+$ IHC positive. In our cohort the CEP17 amplicon almost always involves the HER2 but not the TOP2 locus. Overall agreement on HER2/CEP17 ratio (when applying ASCO/CAP guidelines) was only 64\% (9/14 cases) between the institutions. Discrepant ratios varied from 1.1 to 14.3. The HER2/CEP17 co-amplification is not defined in the ASCO/CAP guidelines, and may result in inaccurate HER2-FISH/SISH status, particularly if only the calculated HER2/CEP17 ratio is reported. It is recommended to report separate CEP17 and HER2 signals in complex HER2/CEP17 patterns.
\end{abstract}

DOI: https://doi.org/10.1007/s10549-011-1642-8

Posted at the Zurich Open Repository and Archive, University of Zurich

ZORA URL: https://doi.org/10.5167/uzh-48545

Journal Article

Published Version

Originally published at:

Varga, Z; Tubbs, R R; Wang, Z; Sun, Y; Noske, A; Kradolfer, D; Bosshard, G; Jochum, W; Moch, H; Öhlschlegel, C (2012). Co-amplification of the HER2 gene and chromosome 17 centromere: a potential diagnostic pitfall in HER2 testing in breast cancer. Breast Cancer Research and Treatment, 132(3):925935.

DOI: https://doi.org/10.1007/s10549-011-1642-8 


\title{
Co-amplification of the HER2 gene and chromosome 17 centromere: a potential diagnostic pitfall in HER2 testing in breast cancer
}

\author{
Zsuzsanna Varga $\cdot$ Raymond R. Tubbs $\cdot$ Zhen Wang $\cdot$ \\ Yang Sun · Aurelia Noske · Doris Kradolfer · Giovanna Bosshard • \\ Wolfram Jochum $\cdot$ Holger Moch $\cdot$ Christian Öhlschlegel
}

Received: 5 April 2011/Accepted: 13 June 2011/Published online: 23 June 2011

(C) Springer Science+Business Media, LLC. 2011

\begin{abstract}
Co-amplification of the centromere on chromosome 17 (CEP17) and HER2 can occur in breast cancer. Such aberrant patterns (clusters) on CEP17 can be misleading to calculate the HER2/CEP17 ratio, and thus underreporting of HER2 amplification. We identified 14 breast cancers retrospectively with HER2/CEP17 co-amplification and performed FISH (fluorescence in situ hybridization) with additional chromosome 17 probes (17p11.1-q11.1, 17p11.2-p12, TP53 on 17p13.1, RARA on 17q21.1-3 and TOP2 on 17q21.3-22) to characterize the spanning of the amplicon in these cases. Furthermore, the $H E R 2$ status was analyzed by means of HER2 silver in situ hybridization (SISH) and immunohistochemistry (IHC). The co-amplification of HER2/CEP17 was compared between the three institutions. TP53 was eusomic in all cases, 17p11.2-p12 in 79\% (11/14), whereas 17p11.1-q11.1 showed chromosomal gain in all cases. RARA was amplified in $10 / 14$ cases $(71 \%)$ and TOP2 in $3 / 14$ cases $(21 \%)$. HER2 was amplified with FISH/SISH in all 14 cases. 9/14 tumors were $3+$ IHC positive (64\%) and 3 cases were $2+$ IHC positive. In our cohort the $C E P 17$ amplicon almost always involves the HER2 but not the TOP2 locus. Overall agreement on HER2/CEP17 ratio (when applying ASCO/CAP
\end{abstract}

Z. Varga $(\varangle) \cdot$ A. Noske · G. Bosshard · H. Moch Institute of Surgical Pathology, University Hospital Zurich, Schmelzbergstrasse 12, 8091 Zurich, Switzerland e-mail: zsuzsanna.varga@usz.ch

R. R. Tubbs $\cdot$ Z. Wang $\cdot$ Y. Sun Section of Molecular Oncologic Pathology, Department of Molecular Pathology, Pathology and Laboratory Medicine Institute, Cleveland Clinic Foundation, Cleveland, OH, USA

D. Kradolfer · W. Jochum · C. Öhlschlegel Institute of Pathology, Cantonal Hospital, St. Gallen, Switzerland guidelines) was only 64\% (9/14 cases) between the institutions. Discrepant ratios varied from 1.1 to 14.3. The HER2/ CEP17 co-amplification is not defined in the ASCO/CAP guidelines, and may result in inaccurate HER2-FISH/SISH status, particularly if only the calculated HER2/CEP17 ratio is reported. It is recommended to report separate $C E P 17$ and HER2 signals in complex HER2/CEP17 patterns.

Keywords HER2 - CEP17 - Co-amplification · FISH · Breast cancer

\section{Introduction}

Therapeutic response to Herceptin ${ }^{\text {TM }}$ in HER2 positive breast cancer can be predicted by the HER2 status in routine diagnostic testing, which has been established by IHC and in situ hybridization (FISH/SISH/CISH) technology [30]. Depending on the applied test, the current diagnostic ASCO/CAP guidelines require different signal values for the evaluation of the HER2 gene status [30]. When using FISH with the CEP17 control, a HER2/CEP17 ratio $>2.2$ is necessary. If FISH, SISH or CISH (chromogenic ISH) is used without a CEP17 control, more than 6 gene copies or clusters of the HER 2 gene are sufficient to determine the HER2 status as positive $[6,7,30]$. If we deal with aberrant patterns, such as clustering of CEP17 and/or $H E R 2$, standard ASCO/CAP criteria for FISH testing can be quite difficult to apply, as exact numeration of CEP17 and HER2 copy signals becomes difficult. Precise algorithm for the interpretation of double clustering is not defined in the ASCO/CAP guidelines. As in co-amplified cases, the HER2/CEP17 ratio per se will be both mathematically and biologically useless if the HER2 signal count becomes nearly the same as the CEP17 signal count. 
Co-amplification of both the HER2 and CEP17 region is a rare event, occurring in less than $1 \%$ of the breast cancer cases tested routinely $[17,23]$.

In this retrospective study, we present a comprehensive analysis of 14 breast cancer cases with cluster forming chromosomal gains on both CEP17 and the HER2 gene by FISH testing. We compared the evaluation of these cases and the standard calculation of the HER $2 / C E P 17$ ratio among the three participating institutes. Large HER2 amplicons can overlap numerous genes on $17 \mathrm{q}$ and $17 \mathrm{p}$. Additionally, we tested the potential amplification status of adjacent genes on $17 \mathrm{q}$ and $17 \mathrm{p}$, including TOP2 (Topoisomerase 2A), RARA (Retinoic Acid Receptor Alpha) and TP53. CEP17 was examined using two different probes covering centromeric and pericentromeric gene sequences of different lengths. Finally, we correlated the amplification status and ratios in all cases to the IHC results of HER2.

\section{Materials and methods}

\section{Study cohort}

Fourteen breast cancer cases with an amplified centromeric region of the chromosome 17 from routine HER2-FISH testing were retrieved from the diagnostic archives at the Institute of Surgical Pathology, University Hospital Zurich, Switzerland and from the Institute of Pathology, Cantonal Hospital St. Gallen, Switzerland. The age of the patients ranged between 39 and 76 years (mean age 61.1 years). All but one tumor corresponded histologically to invasive ductal carcinoma and one case was an intracystic papillary carcinoma with invasive components (Table 1). Seven cases were pT1c, four cases pT2, one case pT3, and two cases pT4. Axillary lymph node metastases were present in 11 patients and the lymph node status was not known for one patient (Nr. 14). Eleven carcinomas were hormone receptor positive. The study was approved by the project review board at the Institute of Surgical Pathology, University Hospital Zurich, Switzerland.

For the study, paraffin blocks of surgical specimens were used in 12 cases and core biopsies in 2 cases.

\section{Methods}

\section{Fluorescence in situ hybridization (FISH)}

Paraffin embedded sections with a thickness of two micrometers were used for all fluorescence in situ hybridization analyses. All procedures for the FISH analyses were carried out by following the recommended protocol of the manufacturers. Probe mixes were hybridized at $37^{\circ} \mathrm{C}$ between 14 and $20 \mathrm{~h}$, washed in Rapid-Wash-Solution I at $73^{\circ} \mathrm{C}$ for $5 \mathrm{~min}$, Rapid-Wash-Solution II and $\mathrm{H}_{2} \mathrm{O}$ for $7 \mathrm{~min}$, air dried and counterstained with DAPI. The reactions were evaluated using an Olympus computer guided fluorescence microscope (BX61, Olympus Schweiz AG, Volketswil, Switzerland). Each case was accompanied by a corresponding hematoxylin and eosin (H\&E) stain in order to identify the invasive tumor component. The HER2 status was analyzed in all 14 cases by the participating institutes (Zurich and Cleveland). Seven of the 14 cases from St.Gallen were tested during the weekly routine FISH

Table 1 Clinico-pathological parameters of the patients

\begin{tabular}{|c|c|c|c|c|c|}
\hline Case Nr. & Age (years) & Histology & TNM stage & ER & PR \\
\hline 1 & 63 & Invasive ductal carcinoma & $\mathrm{pT} 2, \mathrm{pN} 1$ & neg & neg \\
\hline 2 & 71 & Invasive ductal carcinoma & pT3, pN1 & $5 \%$ & $5 \%$ \\
\hline 3 & 73 & Invasive ductal carcinoma & pT4b, pN1 & $60 \%$ & neg \\
\hline 4 & 76 & Invasive ductal carcinoma & $\mathrm{pT} 2, \mathrm{pN} 1$ & $1 \%$ & $1 \%$ \\
\hline 5 & 61 & Invasive ductal carcinoma & pT1c (m), pN1 & $90 \%$ & $80 \%$ \\
\hline 6 & 59 & Invasive ductal carcinoma & pT1c (m), pN1 & neg & $100 \%$ \\
\hline 7 & 53 & Invasive ductal carcinoma & $\mathrm{pT} 4 \mathrm{~b}, \mathrm{pN} 2$ & $30 \%$ & $20 \%$ \\
\hline 8 & 64 & Invasive ductal carcinoma & $\mathrm{pT} 2, \mathrm{pN} 1$ & $40 \%$ & neg \\
\hline 9 & 39 & Invasive ductal carcinoma & $\mathrm{pT} 1 \mathrm{c}, \mathrm{pN} 1$ & $100 \%$ & $90 \%$ \\
\hline 10 & 60 & Invasive papillary carcinoma & pT1c, pN0 & $4 \%$ & neg \\
\hline 11 & 49 & Invasive ductal carcinoma & $\mathrm{pT} 1 \mathrm{c}, \mathrm{pN} 2$ & $14 \%$ & $3 \%$ \\
\hline 12 & 60 & Invasive ductal carcinoma & pT1c, pN0 & $80 \%$ & neg \\
\hline 13 & 74 & Invasive ductal carcinoma & $\mathrm{pT} 2, \mathrm{pN} 1$ & $75 \%$ & neg \\
\hline 14 & 54 & Invasive ductal carcinoma & pT1c, pNx & $85 \%$ & $95 \%$ \\
\hline
\end{tabular}

$E R$ estrogen receptors, $P R$ progesterone receptors. Positivity for hormone receptors is indicated as percentage of positively stained invasive tumor cells 
diagnostics. All other tests were carried out at the Institute of Surgical Pathology, University Hospital Zurich, Switzerland.

\section{HER2 gene}

The HER2 gene was tested by using a dual fluorescence kit (PathVysion $^{\text {TM }}$, Vysis, Abbott AG, Diagnostic Division Baar, Switzerland) containing the HER2 gene (17q11.2$\mathrm{q} 12$, directly labeled with fluorescent spectrum orange) and CEP17 (17p11.1-q11.1, directly labeled with fluorescent spectrum green) (Fig. 2).

\section{CEP17-D17Z1 (centromeric region of chromosome 17)} locus 1

For this locus, a kit labeling the region 17p11.1-q11.1 (D17Z1) of CEP17 (Vysis, Abbott AG, Diagnostic Division Baar, Switzerland) was used (Fig. 2). The probe contained a direct fluorescent labeled area with spectrum aqua.

\section{CEP17-D17S122/HER2 (centromeric region/HER2} gene on chromosome 17) locus 2

For this locus, the probe D17S122, covering the region 17p11.2-p12, was used (Fig 2). The area was visualized by direct fluorescent labeling containing spectrum green. The reactions were carried out on all 14 cases at the Section of Molecular Pathology, Cleveland Clinic. All probes for this locus (along with HER2) were graciously provided by Dr. Robert Jenkins of the Mayo Clinic (Rochester, Minnesota, USA). Spectrum green labeled D17S122-1 (RP11-465O5), D17S122-2 (RP11-726O12), D17S122-3 (RP11-924A14) and D17S122-4 (RP11-136M15). Spectrum orange labeled HER2 BAC1 (RP11-94L15), and HER2 BAC2 (CTD$2019 \mathrm{C} 10)$. All these probes were used to generate the HER2/D17S122 probe cocktail. HER2 was labeled with spectrum orange and D17S122 was labeled with spectrum green. The slides were probed with $1 \mu \mathrm{l} H E R 2$ probe, $2 \mu 1$ D17S122 probe, $2 \mu \mathrm{l}$ human placenta DNA and $5 \mu \mathrm{l}$ hybrisol and incubated overnight at $37^{\circ} \mathrm{C}$.

\section{RARA gene}

For the RARA locus, a combined probe of $L S I^{\circledR} P M L / R A R A$ (Vysis, Abbott AG, Diagnostic Division Baar, Switzerland) was applied. The $R A R A$ gene (17q21.1-q21.3) was directly labeled with the fluorescent spectrum green probe, the $L S I^{\circledR} P M L(15 \mathrm{q} 22$, not assessed in the study) was directly labeled with fluorescent spectrum orange (Fig. 2).
TOP2 gene

For the TOP2 gene, a triple probe (Vysis, Abbott AG, Diagnostic Division Baar, Switzerland) was applied containing the HER2 gene (17q11.2-q12, labeled with spectrum green), the centromere CEP17 (17p11.1-q11.1, labeled with spectrum aqua) and the TOP2 gene (17q21.3q22, labeled with spectrum orange) (Fig. 2).

\section{TP53 gene}

A dual probe (Vysis, Abbott AG, Diagnostic Division Baar, Switzerland) was used for the TP53 gene, containing the TP53 gene (17p13.1, labeled with fluorescent spectrum orange) and CEP17 (17p11.1-q11.1, labeled with fluorescent spectrum green) (Fig. 2).

\section{Silver-enhanced in situ hybridization (SISH) for HER2 and CEP17}

In seven cases (Nr. 1-7) from the Zurich cohort, the HER2 status was also investigated with a silver enhanced in situ hybridization dual probe (Inform, Ventana Medical Systems, Tucson, AZ, USA).

The HER2 DNA probe (catalog Nr.: 780-4332) was directly labeled with silver and the chromosome 17 probe (catalog Nr.: 780-4331) was labeled with red. The signals were detected with the ultraView SISH detection kit and the ultraView red ISH detection kit. The whole process was completely automated using Ventana's Benchmark autostainers according to the manufacturer's protocol. A corresponding $\mathrm{H} \& \mathrm{E}$ control slide was available in each case for the SISH analysis.

\section{Immunohistochemistry for HER2}

Paraffin-embedded sections with a thickness of $2 \mu \mathrm{m}$ were used for the immunohistochemistry. Detection of the HER2 protein was performed with the Ventana Benchmark automated staining system using Ventana reagents (Ventana Medical Systems, Basel, Switzerland) for the entire procedure. Primary antibodies were detected using the iVIEW DAB detection kit and the signal was enhanced using the amplification kit. The following marker was used: Pathway anti-HER2, 4B5 (Ventana, Basel, Switzerland; ready to use without further dilution; concentration, $6 \mu \mathrm{g} / \mathrm{ml})$.

Guidelines used to interpret the in situ hybridization (FISH and SISH) in CEP17 and HER2

The ASCO/CAP guidelines were used to interpret the signals in the FISH and SISH analyses [12, 30]. The 
number of signal copies for $C E P 17$ and HER2 was calculated for each probe. Optical not separable clusters were set to 16 copies in the FISH analyses. Furthermore, the ratios of the dual probes were evaluated. SISH small clusters were set to 6 copies and larger clusters to 12 copies. Similarly, a ratio $>2.2$ was set as an amplified status and ratios $<1.8$ were negative. We used definitions from recently published recommendations by Vance et al. when we were dealing with intratumoral heterogeneity: at least 2 (and up to 4) representative fields from the invasive areas were evaluated. If more than $50 \%$ of the invasive tumor cells in these areas had a HER2/CEP17 ratio higher than 2.2 , we considered the tumor as amplified. We used these criteria to examine all the gene regions named above: HER2, CEP17, RARA, TOP2, and TP53 [16, 25, 27].

The counting and interpretation of the FISH-HER2 signals were performed individually at each institute $(\mathrm{ZV}$, GB, RT, ZW, YS, DK, CO). Signals for SISH-HER2 and FISH-TOP2 were analyzed and counted in Zurich (ZV). The reading and counting of FISH-CEP17 (1), FISH-TP53 and FISH-RARA signals were carried out both in Zurich $(\mathrm{ZV})$ and in St.Gallen (DK, CO). Finally, the evaluation of the FISH-D17S122/HER2 signals was performed in Cleveland (RT, ZW, YS).

\section{Guidelines for the interpretation of the HER2 immunohistochemistry}

The ASCO/CAP guidelines were used to interpret the staining of the HER2 protein expression and scored as follows: 0 (no staining), $1+$ (weak and incomplete membrane staining), $2+$ (strong, complete membrane staining in less than $30 \%$ of the invasive tumor cells or weak/ moderate heterogeneous complete staining in more than $10 \%$ of the invasive tumor cells), and $3+$ (strong complete homogenous membrane staining in more than $30 \%$ of the invasive tumor cells) $[12,30]$.

\section{Interpretation of HER2 Status by HER2/CEP17 ratios}

Although both the HER2 gene and the CEP17 region exhibited 'amplification' separately in each case, there was a huge discrepancy regarding the exact HER2 status of these tumors (Table 3 ). In 5 of 14 cases (35\%) the ratios ranged from 1.1 to 14.3 between the three institutions. These problematic cases included 2 tumors with an immunoreactivity of $3+$ and 3 tumors with an immunoreactivity of $2+$. In 9 of 14 cases $(64 \%)$ the institutions reached an agreement on the HER2 status as amplified or non-amplified, even though the individual ratios varied. We used the criteria mentioned above in the guidelines for the in situ hybridization for all the gene regions $[16,25,27]$. In one institute, the exact method of counting required an electronic excel data sheet as described in previous publications $[16,25,27]$. The other two institutions used direct counting on the computer screen and/or on the fluorescence microscope. Discrepant signal interpretation was principally due to the choice of either reporting the HER2/CEP17 ratio or to reporting the raw signal data. For example, counting 60 cells (as happened in case Nr. 8) showed 1037 HER2 and 1007 CEP17 signals. The HER2 gene count of 17.28 would imply amplification, whereas the CEP17 gene count of 16.78 would mean high chromosomal gain using the recommendations suggested by Viale et al. in his discussion for real polysomic cases $[16,25,27]$. According to the ASCO/CAP guidelines on the other hand, this case is classified as non-amplified as the HER2/CEP17 ratio is 'only' $1.03[12,30]$.

\section{Results}

In situ hybridization

\section{HER2-FISH}

In six cases (Nrs. 4,8,10,11,12,14) we found multiple large clusters, in four cases (Nrs. 1, 2, 5, 7) clusters and gene copies $(>5$ ), in four cases (Nrs. 3, 6, 9, 13) multiple copies of the HER2 gene were present ( $>5$, up to 20-25 gene copies) (Figs. 1, 3a, 4a, b; Tables 2, 3).

\section{HER2-CEP17 SISH}

Seven cases (case Nrs. 1-7) were tested with SISH. In all 7 cases, the HER2 gene was present either in $>5$ copies and/ or in large clusters (100\%) (Fig. 3c). In 3 of 7 cases, both HER 2 and $C E P 17$ were present in clusters (Nrs. 1, 2, 4). In cases Nr. 5 and Nr. 7, CEP17 and the HER2 gene showed large cluster formations as well as multiple gene copies (up to 8 gene copies). In case Nr. 6 both CEP17 and HER2 displayed multiple gene copies (CEP17 up to 8 copies and the $H E R 2$ gene up to 25 copies). Case Nr. 3 showed $>5$ HER2 gene copies (5-8) and up to 8 CEP17 copies.

\section{CEP17 (locus 1)}

CEP17 (locus 1) was analyzed both with a dual HER2 probe as well as with a single probe.

CEP17 (locus 1) dual probe:

All 14 cases (100\%) revealed cluster formation and/or multiple copies of $C E P 17$ (locus 1) (Fig. 1, Table 2). In 6 cases (Nrs. 4, 5, 9, 10, 12, 13) CEP17 was visualized as solitary large clusters (Fig. 3d, 4c). In another 6 cases (Nrs. 1, 2, 7, 8, 
Fig. 1 Graphical representation of amplified gene regions on chromosome 17. Amplification is meant as absolute gene copy number of at least 6 or the presence of clusters in more than $50 \%$ of the tested invasive tumor cells
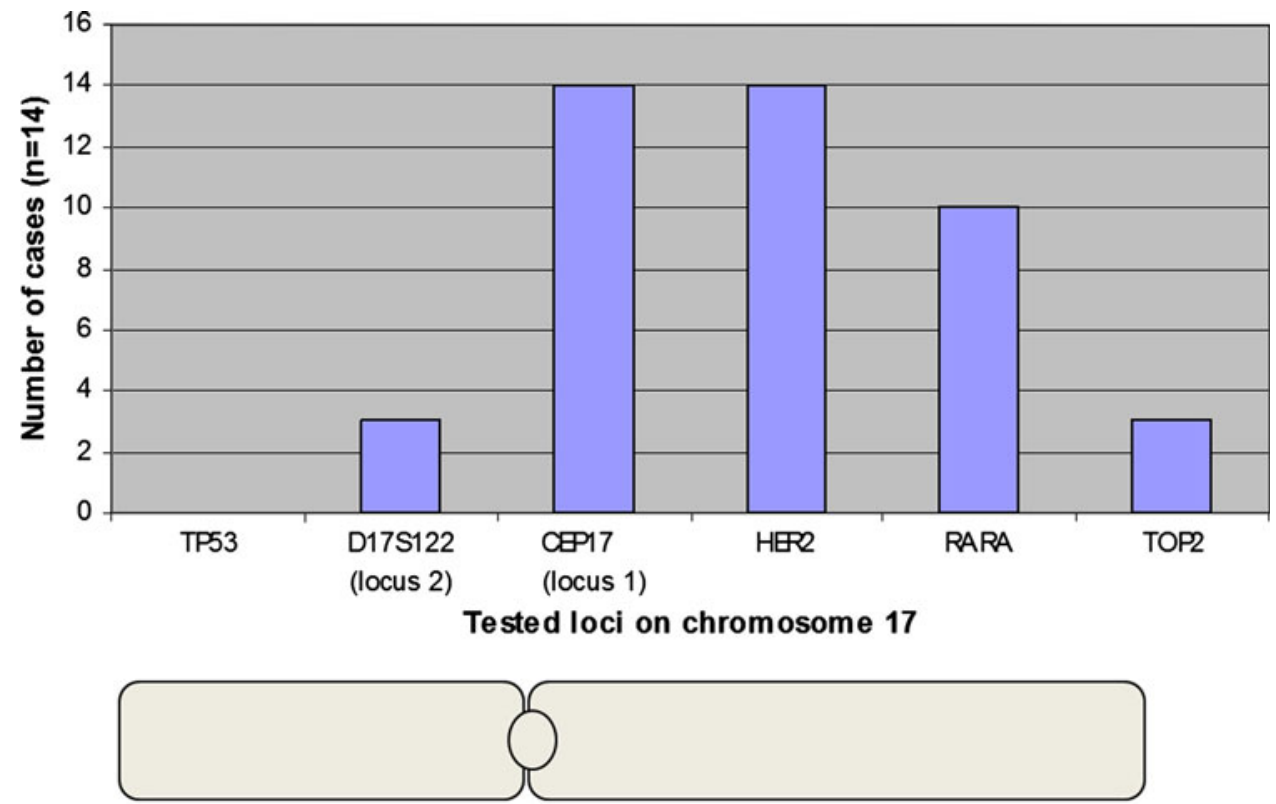

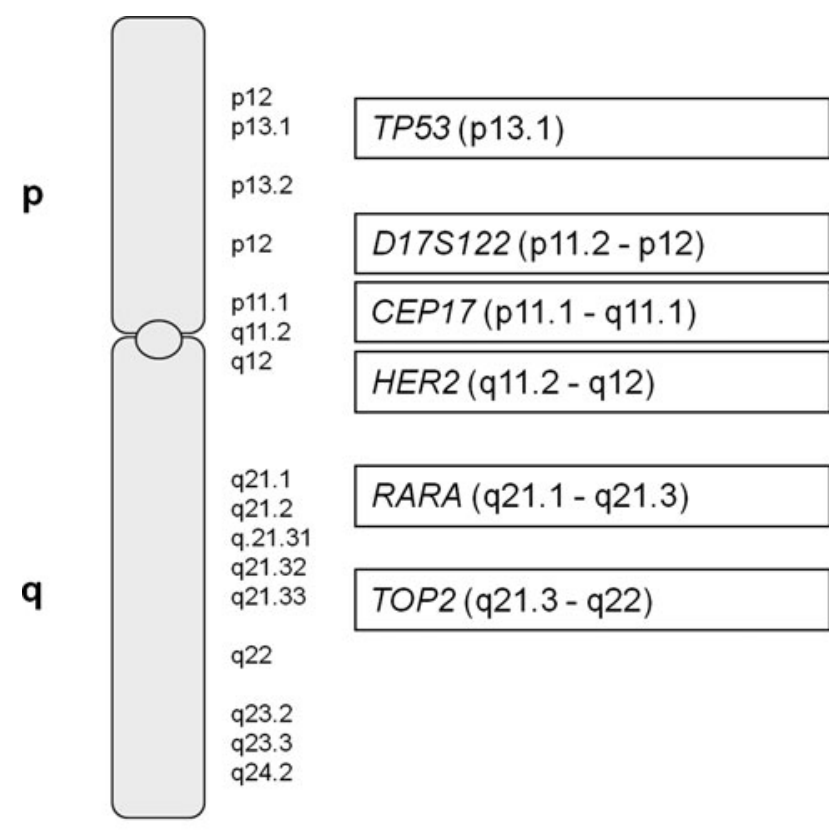

\section{Chromosome 17}

Fig. 2 Anatomical portrayal of the investigated gene loci on chromosome 17

$11,14)$, we found both clusters and up to 8 gene copies. Two cases (Nrs. 3, 6) revealed up to 8-12 gene copies.

\section{CEP17 (locus 1) single probe}

Case Nr. 12 could not be evaluated for this region with the single probe, as no clear signals could be achieved after repeated testing. Large clusters were seen in 3 cases (Nrs. 1, 2, 4). In 4 cases (Nrs. 5, 7, 8, 14), CEP17 was found in clusters and up to 8 gene copies. Two cases (Nrs. 3, 6) revealed up to 8 gene copies and in case Nrs. 9, 10, 11, 13 there were small clusters.

\section{D17S122/HER2 (locus 2)}

Three of 14 cases (21\%) showed multiple copies of D17S122 (locus 2) (Table 2). The average number of copies was 5.5 (Nr. 3), 3.1 (Nr. 6) and 7.1 (Nr. 7). HER2 was present with $>5$ copies in 9 of 14 cases and with $<5$ copies in 5 of 14 cases (Fig. 1).

\section{RARA gene}

Ten of 14 cases $(71 \%)$ revealed amplification of the $R A R A$ gene (Table 2; Figs. 1, 3b). In 2 cases (Nrs. 9 and 10), amplification was a focal finding (at least in 60 cells) and in all the other cases, tumor cells were diffusely amplified. In 2 cases (Nrs. 1, 4), large clusters and up to 8 gene copies were detected. In 5 cases (Nrs. 7, 8, 9, 10, 14), RARA was present in large clusters, and in 3 cases (Nrs. 5, 6, 11) in multiple copies (up to 8). Case Nr. 12 could not be analyzed with this probe as no clear signals were visible.

TOP2 gene

Three of 14 cases $(21 \%)$ showed an amplified TOP 2 region with up to 8 separate gene copies visible in two cases 

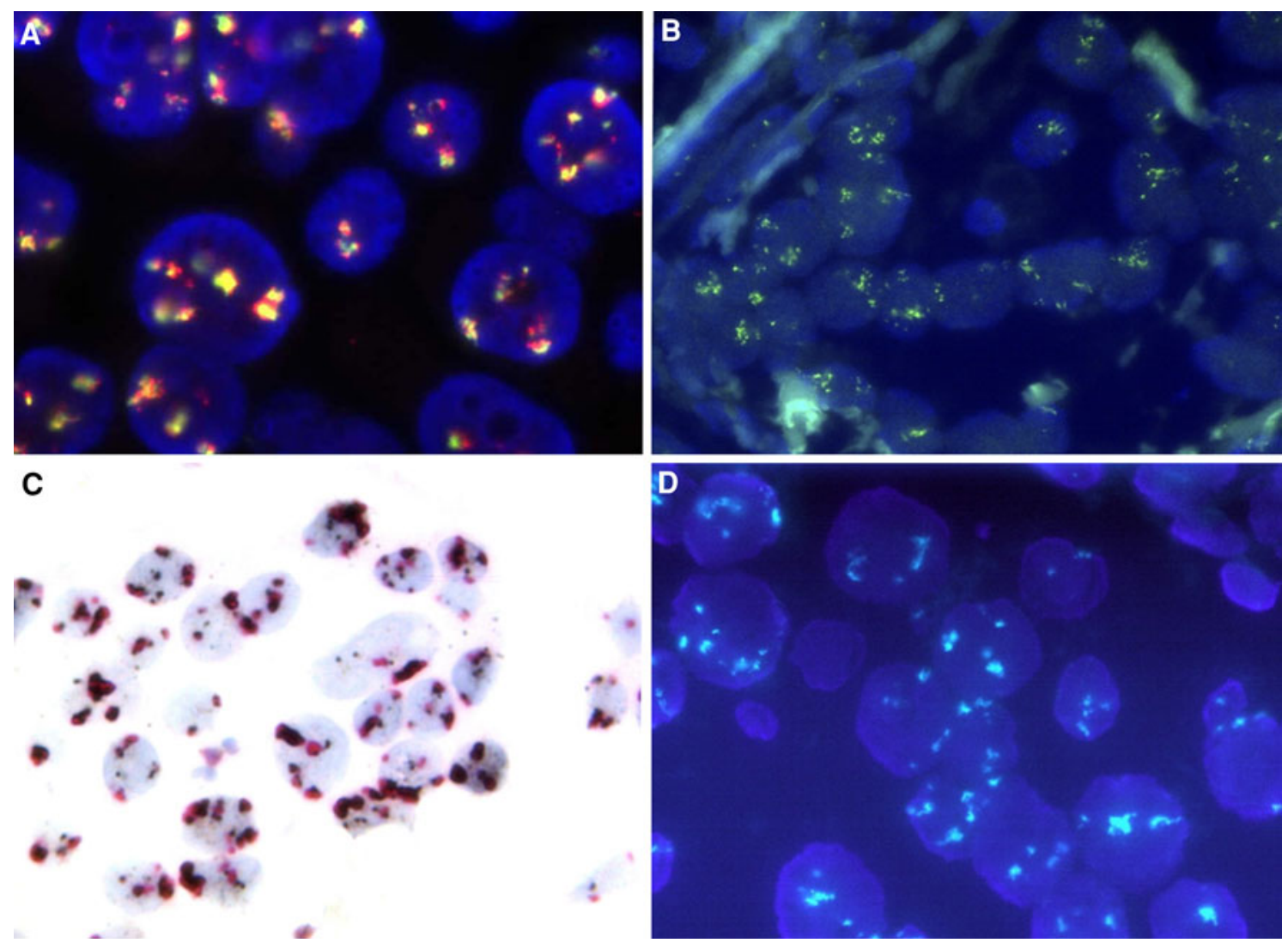

Fig. 3 Different amplification patterns in cluster forming chromosomal gain on 17q. a FISH HER2/CEP17 dual probe (Vysis): HER2: orange, CEP17: green, both are in partially overlapping clusters. b FISH RARA/LSI ${ }^{\circledR} P M L$ dual probe (Vysis): RARA clusters in green

coloration, LSI ${ }^{\circledR}$ PML not photographed. c SISH HER2/CEP17 dual probe (Ventana), HER2: black, CEP17: red, both genes display large overlapping clusters. d FISH CEP17 single probe (Vysis): CEP17: in spectrum aqua, in large clusters
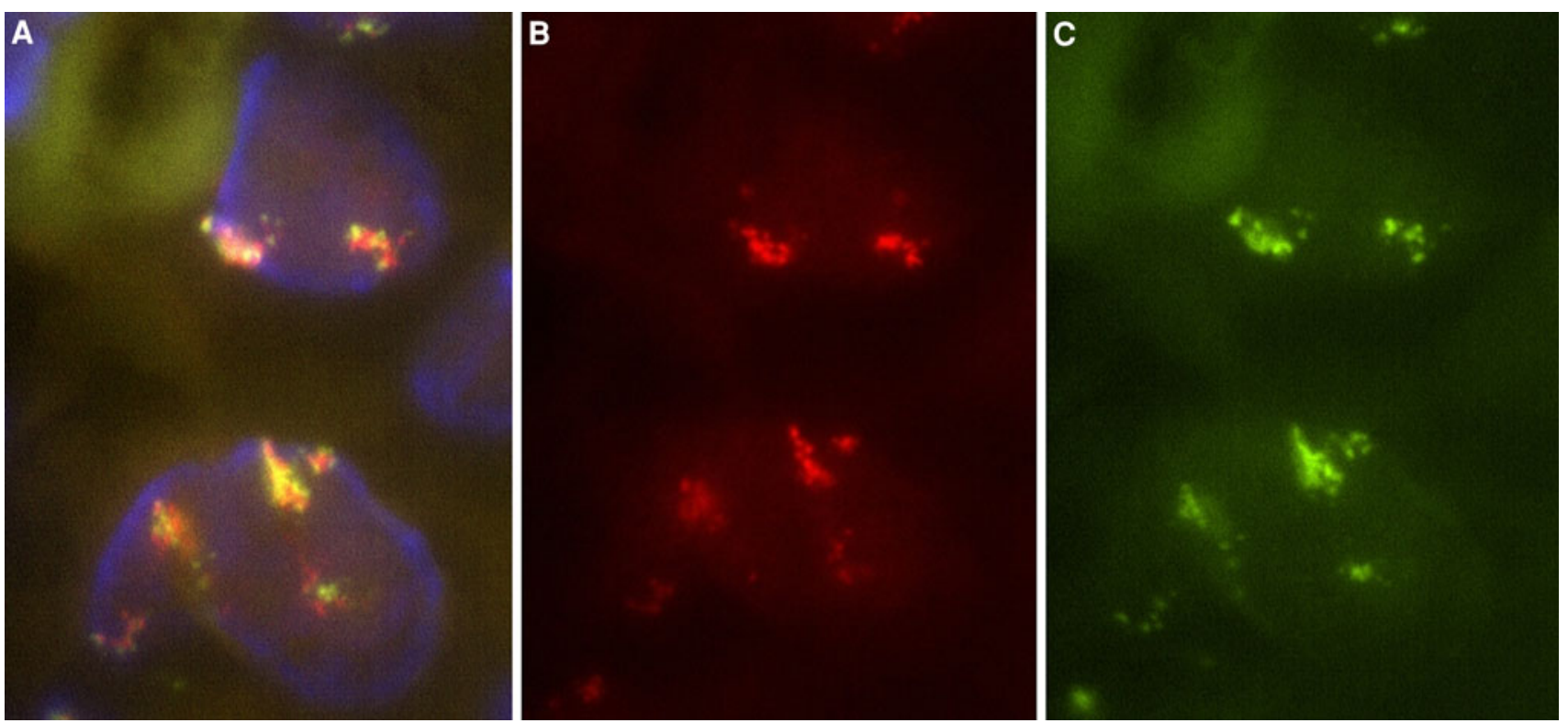

Fig. 4 High magnification of $H E R 2 / C E P 17$ amplicons in the same two carcinoma cells: a FISH HER2/CEP17 dual probe (Vysis): HER2: orange, CEP17: green, both signals in partially overlapping clusters. b FISH HER2 (dual probe with CEP17) (Vysis): HER2: orange in large clusters, $C E P 17$ is switched out. c FISH CEP17 (dual probe with HER2) (Vysis): CEP17: green in large clusters, HER2 is not photographed 
Table 2 Summary of the fluorescence in situ hybridization (FISH) on the tested loci on chromosome 17. Numbers indicate number of cases (total $n=14$ )

\begin{tabular}{llcccc}
\hline & Probe (locus) & Eusomic gene & $\begin{array}{l}\text { Clusters and/ } \\
\text { or gene copies }>5\end{array}$ & $\begin{array}{l}\text { Intratumoral } \\
\text { heterogeneity }\end{array}$ \\
\hline TP53 & $17 \mathrm{p} 13.1$ & 14 & 0 & 0 & $\begin{array}{c}\text { No signals } \\
\text { D17S122 (locus 2) }\end{array}$ \\
CEP17 (locus 1) & $17 \mathrm{p} 11.2-\mathrm{p} 12$ & 11 & 3 & 0 & 0 \\
HER2 & $17 \mathrm{p} 11.1-\mathrm{q} 11.1$ & 0 & 14 & 3 & 0 \\
RARA & $17 \mathrm{q} 11.2-\mathrm{q} 12$ & 0 & 14 & 3 & 0 \\
TOP2 & $17 \mathrm{q} 21.1-\mathrm{q} 21.3$ & 3 & 3 & 3 & 0 \\
\hline
\end{tabular}

CEP17 gene status refers to the whole cohort (including summarized results both with a dual as well as with the single probe)

Table 3 Differential calculation of HER2/CEP17 ratios in the participating institutions. IHC: Immunohistochemistry. FISH: fluorescence in situ hybridization

\begin{tabular}{|c|c|c|c|c|c|}
\hline Case Nr. & HER2 IHC & $\begin{array}{l}\text { Ratio: } H E R 2 / C E P 17 \\
\text { FISH } \\
\text { Institution I }\end{array}$ & $\begin{array}{l}\text { Ratio: } H E R 2 / C E P 17 \\
\text { FISH } \\
\text { Institution II }\end{array}$ & $\begin{array}{l}\text { Ratio: } H E R 2 / C E P 17 \\
\text { FISH } \\
\text { Institution III }\end{array}$ & $\begin{array}{l}\text { Diagnostic } \\
\text { concordance }\end{array}$ \\
\hline 1 & $3+$ & $>2.2$ & 7.6 & Not done & Yes \\
\hline 2 & $3+$ & $>2.2$ & 10.7 & Not done & Yes \\
\hline 3 & 0 & 1.0 & 0.4 & Not done & Yes \\
\hline 4 & $3+$ & $>2.2$ & 17.3 & Not done & Yes \\
\hline 5 & $3+$ & $>2.2$ & 5.4 & Not done & Yes \\
\hline 6 & $3+$ & $>2.2$ & 6.3 & Not done & Yes \\
\hline 7 & $3+$ & $>2.2$ & 2.8 & Not done & Yes \\
\hline 8 & $3+$ & $>2.2$ & 14.3 & 1.1 & No \\
\hline 9 & $2+$ & $<1.8$ & 2.5 & 0.55 & No \\
\hline 10 & $3+$ & $>2.2$ & 1.0 & 1.28 & No \\
\hline 11 & $3+$ & $>2.2$ & 13.4 & 2.89 & Yes \\
\hline 12 & $2+$ & $>2.2$ & 1.3 & 1.16 & No \\
\hline 13 & $1+$ & $<1.8$ & 1.2 & 0.96 & Yes \\
\hline 14 & $2+$ & $>2.2$ & 8.4 & 1.9 & No \\
\hline Positive HER2 status & $9 / 14(64 \%)$ & $11 / 14(78 \%)$ & $10 / 14(71 \%)$ & $1 / 7(14 \%)$ & \\
\hline Disconcordant cases & & & & & $5 / 14(35 \%)$ \\
\hline
\end{tabular}

(Nrs. 1, 14), and in case Nr. 8 large clusters were visible (Fig. 1; Table 2). The CEP17 (locus 1) gene status, analyzed along with $T O P 2$, was found to be identical to the CEP17 gene status analyzed with the HER2/CEP17 dual probe.

\section{TP53 gene}

We could not identify any cases with TP53 amplification or chromosomal gain (Fig. 1; Table 2). All 14 cases revealed 2-3 gene copies of this gene $(0 / 14)$, indicating that there are no cases with a high chromosomal gain in our cohort. As the dual probe also contained the CEP17 region, this was also assessed. We found a $100 \%$ concordance when compared to the dual HER2/CEP17 (locus 1) probe, pointing to an amplified $C E P 17$ (locus 1) in all cases.

\section{HER2 immunohistochemistry}

Nine of 14 cases (64\%) displayed score 3 membranous stains (Table 3). Eight of these were also FISH positive (89\%). One 3+ tumor (Nr. 10) displayed scattered areas (hotspots with at least 60 cells) containing amplified cells with FISH. Three of 14 cases were scored as $2+(21.5 \%)$ and all these $2+$ cases were problematic in the FISH analysis, as the ratio calculation (HER2/CEP17) resulted in diverging values. One case ( $\mathrm{Nr}$. 13) was scored as $1+$ (7\%). This case had a heterogenous pattern between the HER2-IHC and the HER2-FISH as only scattered areas (hotspots of at least 60 cells) were amplified with FISH. This case (Nr. 13) had up to 6 HER2 gene copies and small CEP17 clusters. If counting the absolute HER2 gene copy number, 6 gene copies would qualify this case as amplified. 
Calculating the HER2/CEP17 ratio (ratios: 1.2, 1.8 and 0.96 , respectively) (Table 3 ), this resulted in a nonamplified status. One case (Nr. 3) showed a negative immunostaining $(7 \%)$. This case had a minimum of 8 HER 2 and 8 CEP17 gene copies. The absolute HER2 gene copy number would be sufficient for a positive HER2 status. On the other hand, calculating the HER2/CEP17 ratio ( 0.4 and 1.0 , respectively) results in a negative status.

\section{Discussion}

We identified CEP17/HER2 co-amplification in a series of HER2-FISH assays, and as a result we investigated the nature of the chromosomal region spanning HER2 and CEP17 with different FISH probes to additional loci (TP53, 17p11.1-q11.1, 17p11.2-p12, RARA, and TOP2). We also examined the HER2 gene with SISH and the HER2 protein expression with IHC. The assessment of the co-amplification by FISH was highly diverse in the different laboratories due to missing ASCO/CAP HER2 assay guidelines for this situation. Twelve of 14 cases showing a CEP17/HER2 co-amplification had a HER2 score of either $3+$ or $2+$.

During the last decade the diagnosis of breast cancer has become standardized worldwide by identifying a positive HER2 status by means of immunohistochemistry and FISH [12, 20, 30]. HER2-FISH and HER2 immunohistochemistry assays represent predictive oncologic assays, whereby the staining intensity and gene alterations are characterized by distinct cut-off values of positive signals, and a clear algorithm for the interpretation of the signals exists [12, 20, 30]. Using HER2 test kits approved by the US Food and Drug Administration (FDA), a strong circular membranous stain in more than $30 \%$ of the tested tumor area is defined as a positive HER2 status via immunohistochemistry $[12,30]$. Although a positive HER2 status by means of in situ hybridization has been distinctly defined according to the ASCO-CAP guidelines, there are subtle differences in classifying an amplified status when using different labeling technologies [20,30].

The most widely used assay, the HER2-FISH analysis, requires a ratio $>2.2$ for the copy numbers of $H E R 2$ to CEP17 for an amplified status $[20,30]$. The ASCO/CAP guidelines, however, do not define the role of a chromosomal gain $[20,30]$. A HER2/CEP17 ratio can be misleading in cases showing an extremely high chromosomal gain, as seen in many of our discrepant cases (Figs. 3, 4). On the other hand, the presence of large clusters of the HER2 gene or more than six, respectively, 10 dots of the HER2 gene are sufficient to deal with a positive HER2 status when using silver or chromogenic enhanced in situ hybridization technology (CISH, SISH) [30].
An accurate count of the copy number of the CEP17 region turned out to be quite problematic in our 14 diagnostic HER2-FISH cases, as both the CEP17 region and the $H E R 2$ gene occurred as clusters, to a greater extent as large and to a lesser extent as small clusters. As the exact copy number of both $C E P 17$ and the HER2 gene are required for the assessment of the HER2/CEP17 ratio using ASCO/ CAP criteria, we were confronted by a scenario for which there is no recommendation in the current guidelines [30].

By using SISH technology, however, 5 of the 7 tested cases would easily have qualified as amplified for HER2, as the presence of $H E R 2$ clusters alone adequately fulfill these criteria [30]. Similarly, in a recent paper Marciò et al. [14] showed that $>6$ HER2 gene copies is considered as a therapeutically important amplification, as true CEP17 polysomy very rarely occurs. In this microarray-based comparative genomic hybridization $(\mathrm{aCGH})$ study, Marciò et al. [14] demonstrated that an abnormal CEP17 copy number is most likely due to the amplification of the CEP17 region regardless of the copy number gains of the short and long arms.

As none of our cases showed an amplification of the TP53 gene, but instead a chromosomal gain with 2-3 signals, this indicates that we do not have true polysomic cases in this series. All our cases appear to show large amplicons of the HER2 gene spanning at variable lengths to the centromeric region. Theoretically, it is possible that large HER 2 and CEP17 signals are optically inseparable by using one single bandpass filter.

We compared the interpretation of the cluster formation on CEP17 and the HER2 gene by using the ASCO/CAP guidelines between the three participating institutions and found an enormous discrepancy in the final results. An agreement on the FISH-HER2 status using the HER2/ CEP 17 ratio as negative or positive could only be reached in 9 of 14 cases (64\%) even though cluster formation of the HER 2 gene was present in most cases. The ratios varied between 1.1 and 14.3 in the discrepant cases. This was due to the simple mathematical fact that the CEP17 and HER2 copy numbers were equal or very similar resulting in a practically unusable ratio.

In such situations, following other recommendations, an absolute HER2 gene copy count of $>6$ will fulfill the criteria for a HER2 positive status in all cases (100\%) $[16,25,27]$. Although not included in the ASCO/CAP guidelines, there are papers that propagate their 'own made' criteria, at least for the numeration of the HER2 gene in the case of cluster formation. Tight clustering of the HER2 gene was defined by Simon et al. [21] as being equivalent to 5 gene copies by FISH testing. The classification of small HER2 clusters as being equal to 6 to 10 copies or of large clusters being equal to $>10$ copies in CISH HER2 testing by Tanner et al. [22] seem somewhat 
arbitrary but quite practical if it is about ratio calculation. Then again, several authors avoid defining a random copy number for the HER2 clusters and instead call them 'classical clustering' or admit an 'imprecise signal numeration' as proposed by Lebeau and Sauter $[11,20]$. In co-amplified cases with optically unseparable clusters we set the definition as 16 copies for FISH and as 12 copies (large clusters), respectively, 6 copies (small clusters) for SISH. With this definition, we provided real gene counts, which reflect the amplification status of the cells more realistically than the ratios alone. Luckily enough, most cases showing HER2 gene clustering lack a simultaneous cluster formation of CEP17 enabling an easy diagnostic decision on the HER2 status [11, 20].

According to our knowledge, there is only one study available from 2006 by Troxell et al. on 7 cases with a corresponding editorial from M. Press, addressing the coamplification of CEP17 and the HER2 gene and the question of how to deal with HER2 testing in such settings [17, 23]. Troxell et al. [23] proposed an extended FISH analysis on the neighboring $R A R A$ gene adjacent to the $H E R 2$ gene and also adding a HER 2 immunohistochemistry to the test. Five of their 7 cases were $3+$ positive on a protein level and also exhibited an amplification on the neighboring RARA gene [23].

We took a different approach to characterize the adjacent gene regions in the 14 amplified CEP17 regions. On the one hand, we used a second probe (D17S122) for the CEP17 region, labeling a much shorter DNA sequence (17p11.2-p12) than the one in the Vysis kit (17p11.1q11.1). Cluster formation of CEP17 was detected in 3 of 14 cases with this shorter probe. In one case (Nr. 3) multiple separate signals of CEP17 were seen as well. We extended the adjacent gene regions and additionally tested for the RARA gene, the distally located TOP 2 gene on the long arm, and the TP53 gene on the short arm of chromosome 17. No amplification was detected for the TP53 gene in any of the 14 cases, which corroborates with literature data. TP53 mutations but no amplifications have been found in sporadic HER2 positive breast cancer [29]. In a recent study, TP53 protein overexpression was only detected in unamplified CEP17 polysomic breast cancer cases [10]. The high frequency of RARA co-amplification in our study (10 of 14 cases, $71 \%$ ) is very similar to what has already been reported $[14,23]$. Co-amplification of the RARA/ TOP2/HER2 chromosomal regions can occur in other malignancies as well, as was recently reported in a case of acute myeloid leukemia [2]. It may be true that the TOP2 gene is virtually always co-amplified with the HER2 gene in breast cancer, nevertheless, co-amplification frequencies varying between 30 and $100 \%$ has been published $[9,15,18]$. Therefore, the low TOP2/HER2 co-amplification ratio $(21 \%)$ in our study probably represents a case selection bias.
In our study, all cases showed a HER2/CEP17 co-amplification. Amplification of $C E P 17$ without involvement of the HER2 gene has been reported in the literature. Marchio et al. [14] analyzed 5 cases with an amplified CEP17 region, however, only one of these cases exhibited $>8$ HER2 copies, classifying this case as non-amplified by the HER2/CEP17 ratio.

The presence of multiple gene amplifications on chromosome 17 is a complex process potentially involving a large HER2 amplicon with further altered telomeric genes such as TOP2, RARA, GRB7, STARD3 [8, 26]. It is very likely that the HER2 gene amplification is the first event in the amplicon formation followed by additional chromosomal changes in the telomeric regions $[8,26]$. Deletions and amplifications of other genes have been shown to bear a predictive value in the response to targeted therapy such as anthracycline in HER2 positive breast cancer [1, 8, 9, 18, 26].

Comparative genomic hybridization studies have shown that the long arm of chromosome 17 is particularly prone to genomic changes. Copy number gains have been identified most frequently on $17 \mathrm{q}$ (57\% prevalence) [5]. The formation of large amplicons and the activation of proto-oncogenes probably occur through classical amplification mechanisms such as double minute formations (extrachromosomal units) and homogenously stained regions (as a component of a chromosome) [4, 19].

Between 1999 and 2009, 5,000 FISH-HER2 analyses were performed in Zurich and St.Gallen. HER2/CEP17 co-amplification was diagnosed in 14 of these cases. Therefore, co-amplification of CEP17 and the HER2 gene is a rare event in breast cancer, occurring in less than $1 \%$ of the tested cases.

Accurate interpretation of increased CEP17 and HER2 copy numbers (6 to 10 copies or clusters) is of enormous importance, as false positive or a negative HER2 status can occur if testing is not done with consequence as well as with the correct calculation of the HER2/CEP17 ratio $[3,13,17,23,24,28]$. If multiple complex genetic alterations are detected on chromosome 17 at routine HER2 testing, then careful evaluation of the HER2 amplicon along with the potentially co-amplified neighboring genes, and additional immunohistochemistry for HER2 is necessary $[17,23]$.

In summary, our data indicate that a complex FISH pattern with HER2/CEP17 co-amplification requires confirmatory HER2 analysis by immunohistochemistry. It is recommended to report raw FISH data, including CEP17 signals and HER2 signals as well as the HER2 gene count as the clinically most relevant FISH parameters. Calculation of the HER2/CEP17 ratio can be misleading as such patterns can easily be categorized as 'chromosomal gain' which can result in a 'false negative' HER2 status. 
Moreover, the testing of chromosomal loci lying far away from the HER2 region, such as TP53, is very helpful in defining or ruling out true polysomy.

Acknowledgments The authors wish to thank Dr. Günter Seile for providing case Nr. 14 to the study and Dr. Adriana von Teichman for critical proof-reading and thorough rewriting of the manuscript. There is no conflict of interest for any of the authors.

\section{References}

1. Arriola E, Marchio C, Tan DS, Drury SC, Lambros MB, Natrajan R, Rodriguez-Pinilla SM, Mackay A, Tamber N, Fenwick K, Jones C, Dowsett M, Ashworth A, Reis-Filho JS (2008) Genomic analysis of the HER2/TOP2A amplicon in breast cancer and breast cancer cell lines. Lab Investig 88:491-503. doi:10.1038/ labinvest.2008.19

2. Asleson AD, Morgan V, Smith S, Velagaleti GV (2010) Amplification of the RARA gene in acute myeloid leukemia: significant finding or coincidental observation? Cancer Genet Cytogenet 202:33-37. doi:10.1016/j.cancergencyto.2010.06.003

3. Bartlett JM, Campbell FM, Mallon EA (2008) Determination of HER2 amplification by in situ hybridization: when should chromosome 17 also be determined? Am J Clin Pathol 130:920-926. doi:10.1309/AJCPSDG53BEANCYE

4. KV Cotran RS, Robbins SL (1994) Robbins pathologic basis of disease. W.B. Saunders Company, Philadelphia

5. Dellas A, Torhorst J, Schultheiss E, Mihatsch MJ, Moch H (2002) DNA sequence losses on chromosomes $11 p$ and $18 \mathrm{q}$ are associated with clinical outcome in lymph node-negative ductal breast cancer. Clin Cancer Res 8:1210-1216

6. Dietel M, Ellis IO, Hofler H, Kreipe H, Moch H, Dankof A, Kolble K, Kristiansen G (2007) Comparison of automated silver enhanced in situ hybridisation (SISH) and fluorescence ISH (FISH) for the validation of HER2 gene status in breast carcinoma according to the guidelines of the American Society of Clinical Oncology and the College of American Pathologists. Virchows Arch 451:19-25. doi:10.1007/s00428-007-0424-5

7. Fritzsche FR, Bode PK, Moch H, Kristiansen G, Varga Z, Bode B (2010) Determination of the Her-2/neu gene amplification status in cytologic breast cancer specimens using automated silverenhanced in situ hybridization (SISH). Am J Surg Pathol 34: 1180-1185. doi:10.1097/PAS.0b013e3181

8. Glynn RW, Miller N, Kerin MJ (2010) 17q12-21-the pursuit of targeted therapy in breast cancer. Cancer Treat Rev 36:224-229. doi:10.1016/j.ctrv.2009.12.007

9. Hicks DG, Yoder BJ, Pettay J, Swain E, Tarr S, Hartke M, Skacel M, Crowe JP, Budd GT, Tubbs RR (2005) The incidence of topoisomerase II-alpha genomic alterations in adenocarcinoma of the breast and their relationship to human epidermal growth factor receptor-2 gene amplification: a fluorescence in situ hybridization study. Hum Pathol 36:348-356. doi:10.1016/j. humpath.2005.01.016

10. Krishnamurti U, Zarineh A, Atem FD, Silverman JF (2011) Correlation of immunohistochemical expression of p53 with unamplified chromosome 17 polysomy in invasive breast carcinoma. Appl Immunohistochem Mol Morphol 19:28-32. doi: 10.1097/PAI.0b013e3181e9bb6f

11. Lebeau A, Deimling D, Kaltz C, Sendelhofert A, Iff A, Luthardt B, Untch M, Lohrs U (2001) Her-2/neu analysis in archival tissue samples of human breast cancer: comparison of immunohistochemistry and fluorescence in situ hybridization. J Clin Oncol 19:354-363
12. Lebeau A, Turzynski A, Braun S, Behrhof W, Fleige B, Schmitt WD, Grob TJ, Burkhardt L, Holzel D, Jackisch C, Thomssen C, Muller V, Untch M (2010) Reliability of human epidermal growth factor receptor 2 immunohistochemistry in breast core needle biopsies. J Clin Oncol 28:3264-3270. doi:10.1200/JCO. 2009.25.9366

13. Ma Y, Lespagnard L, Durbecq V, Paesmans M, Desmedt C, Gomez-Galdon M, Veys I, Cardoso F, Sotiriou C, Di Leo A, Piccart MJ, Larsimont D (2005) Polysomy 17 in HER-2/neu status elaboration in breast cancer: effect on daily practice. Clin Cancer Res 11:4393-4399. doi:10.1158/1078-0432.CCR-04-2256

14. Marchio C, Lambros MB, Gugliotta P, Di Cantogno LV, Botta C, Pasini B, Tan DS, Mackay A, Fenwick K, Tamber N, Bussolati G, Ashworth A, Reis-Filho JS, Sapino A (2009) Does chromosome 17 centromere copy number predict polysomy in breast cancer? A fluorescence in situ hybridization and microarraybased CGH analysis. J Pathol 219:16-24. doi:10.1002/path.2574

15. Nielsen KV, Muller S, Moller S, Schonau A, Balslev E, Knoop AS, Ejlertsen B (2010) Aberrations of ERBB2 and TOP2A genes in breast cancer. Mol Oncol 4:161-168. doi:10.1016/j.molonc. 2009.11.001

16. Ohlschlegel C, Zahel K, Kradolfer D, Hell M, Jochum W (2010) Intratumoral heterogeneity of HER2 status in breast carcinoma. Pathologe 31(Suppl 2):292-295. doi:10.1007/s00292-010-1316-Z

17. Press MF (2006) How is Her-2/neu status established when Her2/neu and chromosome 17 centromere are both amplified? Am J Clin Pathol 126:673-674. doi:10.1309/GM16-C018-06EF-URX7

18. Press MF, Sauter G, Buyse M, Bernstein L, Guzman R, Santiago A, Villalobos IE, Eiermann W, Pienkowski T, Martin M, Robert N, Crown J, Bee V, Taupin H, Flom KJ, Tabah-Fisch I, Pauletti G, Lindsay MA, Riva A, Slamon DJ (2010) Alteration of topoisomerase II-alpha gene in human breast cancer: Association with responsiveness to anthracycline-based chemotherapy. J Clin Oncol. doi:10.1200/JCO.2009.27.5644

19. Reddy KS (2007) Double minutes (dmin) and homogeneously staining regions (hsr) in myeloid disorders: a new case suggesting that dmin form hsr in vivo. Cytogenet Genome Res 119:53-59. doi:10.1159/000109619

20. Sauter G, Lee J, Bartlett JM, Slamon DJ, Press MF (2009) Guidelines for human epidermal growth factor receptor 2 testing: biologic and methodologic considerations. J Clin Oncol 27: 1323-1333. doi:10.1200/JCO.2007.14.8197

21. Simon R, Nocito A, Hubscher T, Bucher C, Torhorst J, Schraml P, Bubendorf L, Mihatsch MM, Moch H, Wilber K, Schotzau A, Kononen J, Sauter G (2001) Patterns of her-2/neu amplification and overexpression in primary and metastatic breast cancer. J Natl Cancer Inst 93:1141-1146

22. Tanner M, Gancberg D, Di Leo A, Larsimont D, Rouas G, Piccart MJ, Isola J (2000) Chromogenic in situ hybridization: a practical alternative for fluorescence in situ hybridization to detect HER-2/ neu oncogene amplification in archival breast cancer samples. Am J Pathol 157:1467-1472

23. Troxell ML, Bangs CD, Lawce HJ, Galperin IB, Baiyee D, West RB, Olson SB, Cherry AM (2006) Evaluation of Her-2/neu status in carcinomas with amplified chromosome 17 centromere locus. Am J Clin Pathol 126:709-716. doi:10.1309/9EYM-6VE5-8F2YCD9F

24. Tubbs RR, Pettay JD, Roche PC, Stoler MH, Jenkins RB, Grogan TM (2001) Discrepancies in clinical laboratory testing of eligibility for trastuzumab therapy: apparent immunohistochemical false-positives do not get the message. J Clin Oncol 19: 2714-2721

25. Vance GH, Barry TS, Bloom KJ, Fitzgibbons PL, Hicks DG, Jenkins RB, Persons DL, Tubbs RR, Hammond ME (2009) Genetic heterogeneity in HER2 testing in breast cancer: panel summary and guidelines. Arch Pathol Lab Med 133:611-612 
26. Vanden Bempt I, Drijkoningen M, De Wolf-Peeters C (2007) The complexity of genotypic alterations underlying HER2-positive breast cancer: an explanation for its clinical heterogeneity. Curr Opin Oncol 19:552-557

27. Viale G (2009) Be precise! The need to consider the mechanisms for CEP17 copy number changes in breast cancer. J Pathol 219:1-2. doi:10.1002/path.2593

28. Vranic S, Teruya B, Repertinger S, Ulmer P, Hagenkord J, Gatalica Z (2011) Assessment of HER2 gene status in breast carcinomas with polysomy of chromosome 17. Cancer 117:48-53. doi:10.1002/cncr.25580

29. Wilson JR, Bateman AC, Hanson H, An Q, Evans G, Rahman N, Jones JL, Eccles DM (2010) A novel HER2-positive breast cancer phenotype arising from germline TP53 mutations. J Med Genet 47:771-774. doi:10.1136/jmg.2010.078113

30. Wolff AC, Hammond ME, Schwartz JN, Hagerty KL, Allred DC, Cote RJ, Dowsett M, Fitzgibbons PL, Hanna WM, Langer A, McShane LM, Paik S, Pegram MD, Perez EA, Press MF, Rhodes A, Sturgeon C, Taube SE, Tubbs R, Vance GH, van de Vijver M, Wheeler TM, Hayes DF (2007) American Society of Clinical Oncology/College of American Pathologists guideline recommendations for human epidermal growth factor receptor 2 testing in breast cancer. J Clin Oncol 25:118-145. doi:10.1200/JCO. 2006.09.2775 